\title{
Korean Society of Gastrointestinal Endoscopy Guidelines for Endoscope Reprocessing
}

\author{
Byoung Kwan Son ${ }^{1}$, Byung-Wook Kim${ }^{2}$, Won Hee Kim ${ }^{3}$, Dae-Sung Myung ${ }^{4}$, Young-Seok Cho ${ }^{5}$, Byung lk Jang ${ }^{6}$ and The Disinfection \\ Management and Conscious Sedation Committee of Korean Society of Gastrointestinal Endoscopy \\ ${ }^{1}$ Department of Internal Medicine, Eulji Hospital, Eulji University School of Medicine, Seoul, ${ }^{2}$ Department of Internal Medicine, Incheon St. \\ Mary's Hospital, College of Medicine, The Catholic University of Korea, Incheon, ${ }^{3}$ Digestive Disease Center, CHA Bundang Medical Center, \\ CHA University, Seongnam, ${ }^{4}$ Department of Internal Medicine, Chonnam National University Medical School, Gwangju, ${ }^{5}$ Department of \\ Internal Medicine, Seoul St. Mary's Hospital, College of Medicine, The Catholic University of Korea, Seoul, ${ }^{6}$ Department of Internal Medicine, \\ Yeungnam University College of Medicine, Daegu, Korea
}

The Korean Society of Gastrointestinal Endoscopy (KSGE) issued guidelines for endoscope reprocessing for the first time in 1995, and the version of the guidelines was updated in August 2009, August 2012, and March 2015. Guidelines for endoscope reprocessing should be revised continuously, because new disinfectants and devices are developed and introduced. The current official version of the KSGE guidelines for endoscope reprocessing is explained herein to assist the reader in understanding the KSGE requirements for cleaning and disinfecting endoscopes. Clin Endosc 2017;50:143-147

Key Words: Endoscopy; Guidelines; Endoscope reprocessing

\section{INTRODUCTION}

The Korean Society of Gastrointestinal Endoscopy (KSGE) issued guidelines for endoscope reprocessing for the first time in 1995, and the version of the guidelines was updated in August 2009, August 2012, and March 2015 (Table 1). Among the sections within the guidelines, the disinfection section is being used as an evaluation standard for hospital certification and quality of endoscopy for cancer screening. The current version of the KSGE guidelines for endoscope reprocessing is explained herein to assist the reader in understanding the KSGE requirements for cleaning and disinfecting endoscopes. These guidelines have been established

Received: February 6, 2017 Revised: February 13, 2017

Accepted: February 13, 2017

Correspondence: Byung Ik Jang

Department of Internal Medicine, Yeungnam University College of Medicine, 170 Hyeonchung-ro, Nam-gu, Daegu 42415, Korea

Tel: +82-53-620-3831, Fax: +82-53-654-8386, E-mail: jbi1@yu.ac.kr

(c) This is an Open Access article distributed under the terms of the Creative Commons Attribution Non-Commercial License (http://creativecommons.org/ licenses/by-nc/3.0) which permits unrestricted non-commercial use, distribution, and reproduction in any medium, provided the original work is properly cited. after a thorough review of previous reports and guidelines ${ }^{1-11}$ by The Disinfection Management and Conscious Sedation Committee, KSGE.

\section{GUIDELINES FOR ENDOSCOPE REPROCESSING}

\section{Pre-cleaning}

(1) Upon completion of endoscopy, immediately wipe the exterior of the endoscope beside the bed using gauze soaked in enzymatic detergent or sterilized distilled water.

Explanation: Enzymatic detergent components may vary among manufacturers, but they are commonly effective in decontaminating an endoscope contaminated with blood, polysaccharides, lipids and biofilm during endoscopy. 
Table 1. Korean Society of Gastrointestinal Endoscopy Guidelines for Endoscope Reprocessing: Update 2015

\section{Pre-cleaning}

(1) Upon completion of endoscopy, immediately clean the exterior of the endoscope beside the bed using gauze soaked in enzymatic detergent or sterilized distilled water.

(2) Place the distal end of the endoscope into detergent solution, aspirate detergent through the air/water and biopsy channels, and then blow air through the endoscope channel. These steps should be repeated to remove residual contamination.

(3) After disconnecting the endoscope from the power source, transfer it to a separately located reprocessing area in a designated container. If the distance to the reprocessing area is far, the container should be enclosed to prevent possible environmental contamination.

\section{Cleaning}

(1) Remove all detachable parts from the endoscope.

(2) Detect if there is a leak.

(3) Clean the endoscope with a detergent solution and thoroughly brush all channels and removable parts.

(4) Clean the parts that cannot adequately be brushed using an ultrasonic cleaner while they are immersed in a detergent solution.

(5) Thoroughly rinse the endoscope and detachable parts with clean water to wash away residual detergent (even in the case of using an automatic reprocessor, cleaning needs to be done manually).

\section{Disinfection}

(1) In the case of manual disinfection, use a basin that is large enough and corrosion-resistant. Completely immerse the endoscope and all removable parts completely in a basin of high-level disinfectants; ensure that all channels are completely filled with the disinfectant.

(2) When using any disinfectant solution, follow the manufacturer's instructions for disinfection conditions and time.

(3) If an automatic reprocessor is used, follow all instructions provided in the User's Guide.

\section{Rinse}

(1) Thoroughly rinse the endoscope and flush all channels using clean, drinkable water.

\section{Drying}

(1) Flush each channel with compressed air and $70 \%$ to $90 \%$ ethyl or isopropyl alcohol to facilitate drying.

\section{Storage}

(1) Store endoscopes in a designated, well-ventilated area. When hanging endoscopes in a vertical position, ensure that their distal end does not touch the floor.

\section{Endoscopic accessories}

(1) Disinfect all reusable endoscopic accessories.

(2) Immerse dismantled endoscopic accessories in a detergent solution, and use a brush and sponge to clean the internal surface.

(3) Clean accessories using an ultrasonic cleaner in a detergent solution.

(4) After rinsing accessories with clean water, dry them with a clean cloth and compressed air.

(5) Before storage, sterilize or disinfect accessories, according to the type of accessories and the corresponding manufacturer's instructions. Reusable accessories, such as suction, air water valve, and rubber biopsy valve caps, should receive high-level disinfection before reuse.

(6) Do not use disposable needles or forceps more than once.

\section{Water bottle and connecting tube}

(1) Sterilize the water bottle and its connecting tube once daily and fill with sterile water.

(2) After placing the distal end of the endoscope into detergent solution, aspirate a large volume of detergent solution through the air/water and biopsy channels and then blow air through the endoscope channel. These steps should be repeated to remove residual contamination.

Explanation: One can clean a contaminated endoscope more efficiently by alternating aspiration of the detergent solution and blowing air through the endoscope channel than just aspirating the detergent solution, such as enzymatic or neutral detergent or distilled water. If this pre-cleaning process is not performed immediately after completion of en- doscopy, debris, such as blood and secretion, will lead to clogs within the channel, which cannot be removed completely even through the subsequent disinfection process. When the pre-cleaning step is finished, disconnect the endoscope from the power outlet.

(3) Then, transfer the endoscope to the reprocessing area, which is separated from the procedure room, to a designated container. If the reprocessing area is not nearby, the container should be enclosed to prevent environmental contamination. 
Explanation: The reprocessing area should be physically separate from the procedure room, if possible, and equipped with adequate reprocessing equipment. Good ventilation is essential in the reprocessing area to prevent personnel from being exposed to the toxicity of disinfectants used for reprocessing. The reprocessing area should be divided into clean and dirty areas to avoid cross contaminating the former. There should be designated and adequate space between contaminated endoscopes and clean ones. To minimize the risks of transmitting infections, place contaminated endoscopes in a designated container, and transport them from the procedure room to a separately located reprocessing room. The transport container should be large enough to prevent damage that may occur if the distal end is coiled tightly. Use an enclosed container or bag if the distance to the reprocessing room is far.

\section{Cleaning}

(1) Disconnect and disassemble all detachable parts from the endoscope.

Explanation: Disconnect and disassemble suction valves, air water valves, and rubber biopsy valve caps for cleaning and disinfection.

(2) Perform leak testing.

Explanation: Perform leak testing to detect any damage to the interior or exterior of the endoscope, according to the manufacturer's instructions. As moisture inside the endoscope can lead to malfunction, function of the water-resistant cap should be inspected through leak testing. Leak testing can be performed manually or with a leak tester. Immerse and soak the endoscope in water and carefully observe if there is a stream of air bubbles coming out of the interior of the endoscope. A continuous stream of air bubbles indicates the presence of a leak. In such cases, repair the endoscope before continuing to clean it. Leak testing should be performed before the disinfection cycle. If a leak is not detected, continue to clean the endoscope. Cleaning an endoscope after failed a leak test will cause further damage. Before cleaning, leak testing must be performed to ensure there are no leaks.

(3) Perform manual cleaning with a cleaning solution; thorough brushing of the channels and other detachable parts is required.

Explanation: After checking that the endoscope is free from damage, clean the exterior of the endoscope with a soft cloth or sponge while it is immersed in a detergent solution. Use brushes that are compatible in size with the channels to clean removable debris completely from the inside of the channels. Use a detergent solution that can effectively penetrate and remove debris formed by protein, fat, carbohydrates, and various chemical salts without harming the scope being cleaned. Enzymatic detergent or medical grade, low-foaming neutral detergent solutions are recommended. Foaming can prevent the detergent solution from contacting the endoscope. A soft cloth or sponge should be used once. If not, ensure it is disinfected or sterilized before reuse. All channels and detachable parts should be brushed.

(4) Clean the parts that cannot adequately be brushed using an ultrasonic cleaner while they are immersed in a detergent solution.

(5) After endoscope cleaning, thoroughly rinse the endoscope and detachable parts with clean water to wash away residual detergent (even in the case of using an automatic reprocessor, cleaning needs to be done manually).

\section{Disinfection}

(1) In case of manual disinfection, use a basin that is large enough and corrosion-resistant. Completely immerse the endoscope and all detachable parts completely in a basin of high-level disinfectants; ensure that all channels are completely filled with the disinfectant.

Explanation: Completely immerse the endoscope and detachable parts in a high-level disinfectant solution and ensure that all channels are filled with the disinfectant solution and that no air pockets remain within the channels. Air pockets can allow microorganisms to survive the high-level disinfection process, making disinfection ineffective. Although most high-level disinfectants are reusable, prolonged and repeated use of the same disinfectants reduces the optimal concentrations and effectiveness of disinfection. Minimum effective concentration of disinfectants should be checked to maintain the level of effective disinfection. If the disinfectant solution fails to meet the minimum concentration in a test conducted before use, dispose it. Additionally, disinfectants should not be used after their expiration date.

(2) When using any disinfectant solution, follow the manufacturer's instructions for disinfectant conditions and time.

Explanation: Use disinfectant solutions approved by the Ministry of Food and Drug Safety in Korea, United States 
Food and Drug Administration, Conformité Européene in Europe, and Japanese Ministry of Health, Labor, and Welfare (including those permitted, reported, or registered).

(3) If an automatic reprocessor is used, follow all instructions provided in the User's Guide.

Explanation: An automatic reprocessor is recommended, because it can perform a standardized disinfection process and reduce personnel exposure to disinfectants.

\section{Rinse}

(1) After high-level disinfection, thoroughly rinse the endoscope and flush all channels using clean, drinkable water.

Explanation: Thoroughly rinse the endoscope and flush all channels of the endoscope using sterile water, because residual disinfectants can cause injury of the skin and mucous membrane. The volume of water needed for adequate rinsing is equal to three times the total area to be rinsed. For instance, to rinse an internal channel, at least $150 \mathrm{~mL}$ of water is required.

\section{Drying}

(1) After reprocessing, flush each channel with compressed air and $70 \%$ to $90 \%$ ethyl or isopropyl alcohol to facilitate drying.

Explanation: As moisture breeds microbial bacteria such as Pseudomonas aeruginosa to multiply, drying is a crucial procedure in reprocessing. Keep flushing with alcohol until the alcohol can be seen at the opposite end of each channel. Alcohol helps residual water evaporate as air flows through the channel. Caution is needed because excessively high air pressure can damage the internal channels of the endoscope.

\section{Storage}

(1) Store endoscopes in a designated, well-ventilated area. When hanging endoscopes in a vertical position, ensure that their distal end does not touch the floor.

Explanation: Decontaminated endoscopes should be stored separately from contaminated endoscopes in a clean, dustfree area. Hang endoscopes in a vertical position in a cabinet while all detachable parts are still removed. Store channels, suction valves, air water valve and water-resistant caps separately from the endoscope to prevent clogs. Make sure that the endoscopes hang freely in a vertical position in a clean, well-ventilated, dust-free cabinet. Keep the cabinet clean by wiping the inside everyday with an eco-friendly disinfectant.

\section{Endoscopic accessories}

(1) Disinfect all reusable endoscopic accessories.

(2) Immerse dismantled endoscopic accessories in a detergent solution, and use a brush and sponge to clean the internal surfaces.

(3) Clean accessories using an ultrasonic cleaner in a detergent solution.

(4) After rinsing accessories with clean water, dry them with a clean cloth and compressed air.

(5) Before storage, sterilize or disinfect accessories, according to the type of accessory and the corresponding manufacturer's instructions. Reusable accessories, such as suction, air water valve, and rubber biopsy valve caps, should receive high-level disinfection before reuse.

Explanation: Endoscopic accessories include all detachable parts of the endoscope and devices inserted into the channels. Major devices include biopsy forceps, the hook wire, guide wire, and balloon dilator. All accessories that penetrate the mucosal membrane are recommended for use only once or sterilized before reuse. Sterilization refers to a process that eliminates and destroys all microorganisms, including spores through physical and chemical means.

(6) Do not use disposable needles or forceps more than once.

Explanation: Proper reprocessing of endoscopic accessories is crucial, but it is also important to consider that repeated sterilization and reuse can gradually reduce the effectiveness of the accessories. In the case of biopsy forceps, repeated reprocessing may cause damage to the forceps, because they are not durable, making them more susceptible to contamination. Biopsy forceps have been reprocessed and reused in a cost-effective way. However, biopsy forceps are increasingly used only once in many countries, and Lim et al. ${ }^{12}$ reported that the clinical relevance of biopsy forceps was greater when they were used only once. Additionally, single-use biopsy forceps can be cost-effective if the reprocessing costs are high. However, disposable biopsy forceps must be used for patients with infectious diseases to prevent the spread of infection. The 
guidelines for using disposable needles are increasing in Europe.

\section{Water bottle and connecting tube}

(1) Sterilize the water bottle and its connecting tube once daily and fill with sterile water.

Explanation: To date, there has been no report of the optimal replacement frequency, safety, and potential risks associated with water bottle, lens wash water, tubing for air insufflation, waste vacuum container, and suction tube used in endoscopy. However, these accessories should be disinfected once daily and contain sterile water.

\section{CONCLUSIONS}

The KSGE guidelines are compiled based on international guidelines to provide global standards for endoscope reprocessing. Compliance with this guidance will minimize the risk of transmission of endoscopy-related infection.

\section{Conflicts of Interest}

The authors have no financial conflicts of interest.

\section{Acknowledgments}

The Disinfection Management and Conscious Sedation Committee, Korean Society of Gastrointestinal Endoscopy greatly appreciate Jinsu Kim (The Catholic University of Korea), Jae-Young Jang (Kyung Hee University), Ja Seol Koo (Korea University College of Medicine), and Jung Ho Park (Sungkyunkwan University) for their sincere devotion to preparing this guideline.

\section{REFERENCES}

1. The Disinfection Management Committee. Sodog-aeg-ui jonglyuwa teugseong [Types and characteristics of disinfectant]. In: The Disinfec- tion Management Committee of Korean Society of Gastrointestinal Endoscopy, ed. Sohwagwan naesigyeong secheog mich sodog-ui giljab-i [Guidelines for disinfection of gastrointestinal endoscopes]. Seoul: Medbook; 2012. p. 23-34.

2. Division of Healthcare Policy. Uilyogigwan sayong-gigu mich mulpum sodog jichim gosi jejeong allim [Guidelines for medical instruments and supplies] [Internet]. Sejong: Ministry of Health and Welfare; c2010 [updated 2012 Jul 12; cited $2017 \mathrm{Feb} 6$ ]. Available from: https://www.mohw. go.kr/front_new/jb/sjb0406vw.jsp?PAR_MENU_ID=03\&MENU_ $\mathrm{ID}=030406 \&$ CONT_SEQ=320872\&page $=1$.

3. Korea Center for Disease Control \& Prevention. Guideline for disinfection and sterilization in healthcare facilities [Internet]. Cheongju: Korea Center for Disease Control \& Prevention; c2014 [updated 2014 Dec; cited 2017 Feb 6]. Available from: https://webcache.googleusercontent. com/search?q=cache:kgWQDwm7ewIJ:cdc.go.kr/CDC/cms/cmsFileDownload.jsp\%3Ffid\%3D136\%26cid\%3D21224\%26fieldName\%3DattachGrp\%26index\%3D10+\&cd=1\&hl=ko\&ct=clnk\&gl=kr.

4. Oh HJ, Kim JS. Clinical practice guidelines for endoscope reprocessing. Clin Endosc 2015;48:364-368.

5. Hookey L, Armstrong D, Enns R, Matlow A, Singh H, Love J. Summary of guidelines for infection prevention and control for flexible gastrointestinal endoscopy. Can J Gastroenterol 2013;27:347-350.

6. ASGE Quality Assurance In Endoscopy Committee, Petersen BT, Chennat J, et al. Multisociety guideline on reprocessing flexible gastrointestinal endoscopes: 2011. Gastrointest Endosc 2011;73:1075-1084.

7. Society of Gastroenterology Nurses and Associates, Inc. SGNA standards: standards of infection control in reprocessing of flexible gastrointestinal endoscopes. Gastroenterol Nurs 2010;33:70-80.

8. Yoon IJ. Tips for safe endoscopic reprocessing. In: The 49th Seminar of Korean Society of Gastrointestinal Endoscopy; 2013 Aug 25; Goyang, Korea. Seoul: Korean Society of Gastrointestinal Endoscopy; 2013. p. 324-327.

9. Son BK. Disinfectants for endoscope reprocessing. In: The 50th Seminar of Korean Society of Gastrointestinal Endoscopy; 2014 Mar 30; Goyang, Korea. Seoul: Korean Society of Gastrointestinal Endoscopy; 2014. p. 327-329.

10. Oh KJ. Reprocessing and maintaining of endoscope accessories. In: The 50th Seminar of Korean Society of Gastrointestinal Endoscopy; 2014 Mar 30; Goyang, Korea. Seoul: Korean Society of Gastrointestinal Endoscopy; 2014. p. 330-334.

11. Cho YS. Importance of endoscope reprocessing and new guideline for reprocessing. In: The 52th Seminar of Korean Society of Gastrointestinal Endoscopy; 2015 Mar 29; Goyang, Korea. Seoul: Korean Society of Gastrointestinal Endoscopy; 2015. p. 338-342.

12. Lim CH, Choi MG, Kim WC, et al. Performance and cost of disposable biopsy forceps in upper gastrointestinal endoscopy: comparison with reusable biopsy forceps. Clin Endosc 2012;45:62-66. 